\title{
Time-Reversible Dynamical Systems for Turbulence
}

\author{
L. Biferale ${ }^{1}$, D. Pierotti ${ }^{2}$ and A. Vulpiani ${ }^{3}$
}

November 18, 2018

\author{
${ }^{1}$ Dipartimento di Fisica, Università di Tor Vergata \\ Via della Ricerca Scientifica 1, I-00133 Roma, Italy and \\ Istituto Nazionale di Fisica della Materia, unità di Tor Vergata \\ 2 Dipartimento di Fisica, Università dell' Aquila \\ Via Vetoio 1, I-67010 Coppito, L'Aquila, Italy and \\ Istituto Nazionale di Fisica della Materia, unità dell'Aquila \\ 3 Dipartimento di Fisica, Università di Roma "la Sapienza" \\ Piazzale Aldo Moro 5, I-00185 Roma, Italy and \\ Istituto Nazionale di Fisica della Materia, unità de "La Sapienza"
}

\begin{abstract}
Dynamical Ensemble Equivalence between hydrodynamic dissipative equations and suitable time-reversible dynamical systems has been investigated in a class of dynamical systems for turbulence. The reversible dynamics is obtained from the original dissipative equations by imposing a global constraint.

We find that, by increasing the input energy, the system changes from an equilibrium state to a non-equilibrium stationary state in which an energy cascade, with the same statistical properties of the original system, is clearly detected.
\end{abstract}

PACS number 47.27.Jv, 47.90.+a, 05.45.+b

To the memory of Giovanni Paladin. 


\section{Introduction}

One of the most important open problem in classical physics is the understanding the statistical features of fully developed turbulence (FDT).

A fully developed turbulent flow is a dissipative system described by the Navier-Stokes (NS) equations in the limit of high Reynolds numbers $(R e)$.

On one hand, direct numerical simulations of turbulent flows are strongly limited due to the huge amount of excited degrees of freedom: a simple argument due to Landau, shows that the number of degrees of freedom, which should be taken into account for a correct description of a turbulent flow, increases as $R e^{9 / 4}$.

On the other hand, analytical attempts to derive the multi-points velocity probability distribution function have repeatedly failed due to the strong coupling regime and due to the highly non-gaussian probability distribution functions (PDF) developed at small scales by the velocity field [1] Phenomenological approaches, or simplified dynamical and deterministic systems, have been therefore often used for studying the mechanisms generating the turbulent energy cascade.

From the analytical point of view, the main obstacle to the possibility of performing the 0-th step toward a theory of turbulence is certainly connected to the strong dissipative and far-from-equilibrium character of $3 \mathrm{~d}$ turbulent flows. In $2 \mathrm{~d}$ turbulence, where energy is almost not dissipated at all, some analytical tools based on quasi-equilibrium statistical ansatz have indeed been developed [2].

Strongly chaotic dynamical systems as the Anosov systems are the only cases where, although being still dissipative and chaotic, analytical tools have been developed with relative success, at least in the case of low-dimensions [3].

Recently [4], Gallavotti and Cohen proposed that a chaotic high-dimensional dynamical system in a stationary state can be regarded as a smooth dynamical system with a transitive Axiom-A global attractor or, if it is timereversible, as a smooth transitive Anosov system, as far as macroscopic properties are concerned. This is the so-called Chaotic Hypothesis.

This hypothesis, as the ergodic hypothesis, can be proved only in very particular systems. Nevertheless, it is interesting to analyze some of its consequences.

Likewise, Gallavotti [5, 6] conjectured a Dynamical Ensemble Equiva- 
lence between some dissipative systems (in this paper we will refer only to 3d Navier-Stokes equations) and their non-equilibrium but time-reversible formulation. Here, equivalent must be meant that the averages of local variables in the two systems, i.e. the original one and its time-reversible formulation, are the same, in a suitable limit. For hydrodynamic dissipative systems this limit is that of FDT, i.e. $R e \rightarrow \infty$.

The reversible dynamics is obtained from the original dissipative equations by imposing a constraint, such as to keep constant in time those macroscopic observables (as the total energy) which would have only had stationary averages in the original systems.

Having a time-reversible system and applying the chaotic hypothesis, some large-deviation properties of the fluctuation of the entropy-production rate in the system can be proved [6].

In Navier-Stokes equations, the reversible dynamics is achieved by introducing a sort of "eddy viscosity" which removes the input energy with perfect efficiency. Viscosity becomes non-positive defined and strongly correlated with the large-scale flow where energy is injected.

As far as we know, the idea of reversible NS equations was introduced for the first time by She and Jackson [7]. They did not exploit global constraints, but imposed that the energy contained in each "momentum shell" was constant.

Let us also mention that a constraint of constant energy has been implemented by using the Kraichnan's eddy-viscosity parameterization [8] in low-resolution large-eddies simulations of NS equations in [9]. With such a parameterization of the viscosity one has very weak fluctuations of the energy (less than 1of high-resolution numerical simulations.

From our point of view, the interest in models with global constraints stems from the possibility to describe a global macroscopic dissipative and irreversible physics starting from a deterministic reversible dynamics. The approach can be seen as a bridge from microscopic reversible dynamics to macroscopic irreversible dynamics and, more interesting, a possible systematic tool for going with continuity from a pure-equilibrium and conservative systems to a strong dissipative and far-from-equilibrium time evolution.

In this paper, we investigate these ideas in Shell Models, i.e. a class of simplified dynamical systems for turbulence (for a recent review see [10], for a tutorial introduction see [11]). In particular, we will analyse in details the smooth transition from the equilibrium system at zero viscosity and zero 
external forcing to a (formally)-reversible systems which possess anyway a non-equilibrium flux of energy from large to small scales.

The paper is organised as follows. In section 2 we briefly review the statistical mechanics of a perfect fluid and the ideas presented by Gallavotti in [ [6, 5. 6] concentrating only to the case of Hydrodynamical systems (NavierStokes eqs.). In section 3 we discuss Shell Models philosophy and we describe Gallavotti's implementation to our case. In section 4 we present our numerical results. Conclusions follow in section 5 .

\section{Equilibrium and Non-equilibrium Statisti- cal Mechanics}

In a $3 D$ perfect fluid, i.e. with vanishing external forcing $\nu=0$, the evolution of the velocity field is given by the Euler equations which conserve two quadratic functional, the kinetic energy and the helicity:

$$
E=\frac{1}{2}\left\langle v^{2}\right\rangle \quad H=\frac{1}{2}\langle\mathbf{v} \cdot \omega\rangle,
$$

where $\omega=\nabla \times \mathbf{v}$ is the vorticity. In this case, it is possible to construct a statistical mechanics as for a gas: by using the conservation laws and the conservation of the volume in phase space one obtains a gaussian distribution.

For simplicity let us start by neglecting the helicity conservation. To be explicit, let us consider an incompressible inviscid fluid in a cube with periodic boundary conditions, so that the velocity field can be expanded in Fourier series as

$$
v_{j}(\mathbf{x})=L^{-3 / 2} \sum_{k} e^{i \mathbf{k} \cdot \mathbf{x}} v_{j}(\mathbf{k})
$$

with $\mathbf{k}=2 \pi \mathbf{n} / L$ and $\mathbf{n}=\left(n_{1}, n_{2}, n_{3}\right)$, where $n_{i}$ are integers. The variables $v_{j}(\mathbf{k})$ are not completely independent, since from the incompressibility condition and the fact that $\mathbf{v}(\mathbf{x})$ is real, it follows that

$$
\mathbf{k} \cdot \mathbf{v}(\mathbf{k})=0 \quad \text { and } \quad \mathbf{v}(\mathbf{k})=\mathbf{v}^{*}(-\mathbf{k})
$$

In any case, it is straightforward to introduce a new set of independent variables $X_{a}$, where now $a$ labels the spatial component and the wave vector. By using an ultraviolet truncation, $\mathbf{v}(\mathbf{k})=0$ for $k>k_{\max }$, and by introducing 
(2) in the Euler equations one obtains a set of ordinary differential equations (ODEs) with the structure

$$
\frac{d X_{a}}{d t}=\sum_{b, c} M_{a b c} X_{b} X_{c}
$$

where $M_{a b c}=M_{a c b}$ and $M_{a b c}+M_{b c a}+M_{c a b}=0$ with $a=1, \cdots, N \sim k_{\text {max }}^{3}$. We stress the fact that the ultraviolet truncation is necessary in order to avoid the infinite energy problems of classical field theory.

It is easy to verify that 2 preserves the volume in the phase space as well as the energy, namely

$$
\sum_{a} \frac{\partial}{\partial X_{a}}\left(\frac{d X_{a}}{d t}\right)=0 \quad \text { and } \quad \frac{d E}{d t}=\frac{1}{2} \frac{d}{d t} \sum X_{a}^{2}=0
$$

These conservation laws are sufficient to construct the probability distribution of the variables $\left\{X_{a}\right\}$ [12]: using the ergodic hypothesis, one obtains the microcanonical probability measure

$$
P_{m}\left(\left\{X_{a}\right\}\right) \sim \delta\left(\frac{1}{2} \sum_{a} X_{a}^{2}-E\right)
$$

It is well known that, in the limit $N \rightarrow \infty$, this is equivalent to the canonical measure

$$
P_{c}\left(\left\{X_{a}\right\}\right) \sim \exp -\left(\frac{\beta}{2} \sum_{a} X_{a}^{2}\right)
$$

where the Lagrange multiplier $\beta$ satisfies the relation

$$
<X_{a}^{2}>=\frac{2 E}{N}=\beta^{-1}
$$

In two dimensions, the helicity $H \equiv 0$ and there exists a second conserved quantity, the enstrophy

$$
\Omega=\frac{1}{2} \int \omega^{2} d^{2} x
$$

which is the mean square vorticity. In terms of the $X$ variables, it can be written as

$$
\Omega=\frac{1}{2} \sum k_{a}^{2} X_{a}^{2}
$$


As a consequence, the microcanonical probability measure in $2 d$ is

$$
P_{m}\left(\left\{X_{a}\right\}\right) \sim \delta\left(\frac{1}{2} \sum_{a} X_{a}^{2}-E\right) \delta\left(\frac{1}{2} \sum_{a} k_{a}^{2} X_{a}^{2}-\Omega\right)
$$

and the corresponding canonical measure is

$$
P_{c}\left(\left\{X_{a}\right\}\right) \sim \exp -\left(\frac{\beta_{1}}{2} \sum_{a} X_{a}^{2}+\frac{\beta_{2}}{2} \sum_{a} k_{a}^{2} X_{a}^{2}\right)
$$

where the Lagrange multipliers satisfy the relation

$$
<X_{a}^{2}>=\frac{1}{\beta_{1}+\beta_{2} k_{a}^{2}}
$$

In 3-d one can repeat a similar argument, taking into account the helicity conservation. In this case, being the helicity non positive defined, one as to assume suitable constraints for the generalized temperature related to the helicity [13].

The above results are, both in $2 d$ and in $3 d$, well reproduced by numerical simulations [12].

The limit $\nu \rightarrow 0$ (equivalent to $R e \rightarrow \infty$ ) is singular and cannot be interchanged with the limit $N \rightarrow \infty$. Therefore, the statistical mechanics of an inviscid fluid has a quite limited relevance on the behaviour of the NavierStokes equations at high Reynolds number. Recently some authors proposed the use of conservative statistical mechanics to justify some behaviours of real fluids, e.g the Jupiter's red spot and the emergence of organized structures [14, 15, 16]. The applicability of this approach is limited to some particular quasi equilibrium two-dimensional situations.

On the other hand, both from phenomenological arguments and experimental results, we know that the statistical mechanics of fully developed turbulence has peculiarities rather different from the usual statistical mechanics of conservative systems. In the limit of FDT the energy fluctuates around its mean value and in addition one has an energy cascade from large to small scales.

The turbulence is described by a dissipative system (essentially a high dimensional truncation of the Navier-Stokes equations with $|\mathbf{k}|<k_{\max }=$ $O\left(R e^{3 / 4}\right)$ in which the volume in the phase space is not conserved. Let us stress again that the two limits $\nu \rightarrow 0$ and $k_{\max } \rightarrow \infty$ must be take in a 
suitable way in order to obtain the correct physical result for the turbulence. If one wants that the mean energy dissipation is $O(1)$ in the limit $R e \rightarrow \infty$ one has to take $k_{\max }>O\left(R e^{3 / 4}\right)$.

In order to have a statistical stationary state one needs two basic ingredients: a 'friction' mechanism and a coupling with an external forcing or 'reservoire'. A typical example of statistical stationary state is given by conductive systems where an external electric field and a friction mechanism, mimicking the electrical resistivity, leads to a macroscopic steady current.

Recently time-reversible and conservative systems have been introduced in the issue of the non-equilibrium statistical mechanics of stationary state. For sake of self consistency we recall one of the simplest system of this class. Let us consider $N$ independent particles of mass $m$, with coordinates and momenta $\mathbf{q}_{i}$ and $\mathbf{p}_{i}$ respectively, on a square domain (in 2 or 3 dimensions) with periodic boundary condition for the variables $\mathbf{q}_{i}$. Using a suitable external potential $V(\mathbf{q})$ we can mimic the elastic scattering with rigid obstacles in order to have basically a "Lorentz gas". The equation of motion are:

$$
\begin{aligned}
\frac{d \mathbf{q}_{i}}{d t} & =\frac{1}{m} \mathbf{p}_{i} \\
\frac{d \mathbf{p}_{i}}{d t} & =-\frac{\partial V}{\partial \mathbf{q}_{i}}
\end{aligned}
$$

The system is chaotic and one can expect the usual microcanonical distribution. Of course there is no net current. In order to have a current in the $x$ direction it is necessary to add in the eq. (14) a term $E \mathbf{e}_{1}$, where $\mathbf{e}_{1}$ is the versor in the $x$ direction. At the same time, if one wants to focus on stationary aspects, some energy-loosing mechanism must be added. Standard phenomenology would suggest the insertion of a viscous irreversible term of the form $-\alpha \mathbf{p}_{i}$ in the equation of motion governing the evolution of momenta. In this way, one is naturally lead to a stationary state.

Recently, in [17], the idea of mimicking this behaviour by means of an exactly conservative and reversible physics has been proposed by using instead of a constant viscous coefficient $\alpha$, a perfect energy-sink, correlated with all scales and able to reabsorb instantaneously all excess of energy injected by the forcing term in the system. This ideal viscosity must acquire an explicit time-dependency and works out as a Lagrange multiplier such as the total energy is an invariant of motion. Being the forcing mechanism not-positive defined, also the ideal viscosity will be not-positive defined. 
The system now develops a net current and all the phenomenology of a dissipative physics. The natural question which arises is how much the original dynamics is preserved by this very-strong perturbation of the equation of motion.

In 17] some numerical simulation of eqs. (3)-(4) have been performed showing that some of the main signature of the original physics are still present in the modified model with the advantage, in the latter, that also some analytical investigation can be carried out. In particular the most important consequence of the chaotic hypothesis is the fluctuation theorem that is an exact parameterless prediction. This theorem concern the probability distribution function of the contraction rate of of the attractor surface element (for a detailed discussion see [6]). Let us note that is very difficult, save for very particular systems, to test this prediction as the attracting sets usually are unknown.

In the next section we will investigate a similar problematic in a class of dynamical systems for turbulent flows, called Shell-Models. In particular, we want to understand how much freedom is allowed in the choice of a viscousmodelization without perturbing too much the main physical framework, and/or quantifying the aspects of the perturbation, eventually.

The goal consists in having a reversible dynamics showing in some limit (to be defined) the same physics of a turbulent dissipative flow.

\section{Time-reversible Shell Models}

One of the most intriguing problems in 3 dimensional turbulence is related to the understanding of the non linear dynamical mechanism triggering and supporting the energy cascade from large to small scales. Following the Richardson scenario that energy should be transferred downwards in scales, Kolmogorov [18 (K41) postulated that the energy cascade should follow a self-similar and homogeneous process entirely dependent on the energy transfer rate, $\epsilon$. This idea, with the assumption of local isotropy and universality of the small scales, eventually led to a precise prediction:

$$
S_{p}(l) \equiv\left\langle(\delta v(l))^{p}\right\rangle=C_{p}\left\langle(\epsilon(l))^{p / 3}\right\rangle l^{p / 3}
$$

where $C_{p}$ are constants and $\epsilon(l)$ is the coarse-grained energy dissipation over a scale $l$ supposed to be in the . inertial range, i.e. much smaller than 
the integral scale and much larger than the viscous dissipation cutoff. If $S_{p}(l) \sim l^{\zeta(p)}$ and $\left\langle\epsilon^{p}(l)\right\rangle \sim l^{\tau(p)}$ then

$$
\zeta(p)=p / 3+\tau(p / 3)
$$

In K41 the $\epsilon(l)$ statistic is assumed to be $l$-independent, or $\tau(p)=0$, implying $\zeta(p)=p / 3, \forall p$, in particular $\zeta(2)=2 / 3$ or, equivalently, the energy spectrum going as $k^{-5 / 3}$. On the other hand, there are many experimental and numerical [19, 20, 21, 22] results telling us that K41 scenario for homogeneous and isotropic turbulence is quantitatively wronged. Strong intermittent bursts in the energy transfer have been observed and non trivial $\tau(p)$ set of exponents measured.

Shell models have demonstrated to be very useful for the understanding of many properties connected to the non-linear turbulent energy transfer 23.[30]. The most popular shell model, the Gledzer-Ohkitani-Yamada (GOY) model ([23]-[30]), has been shown to predict scaling properties for $\zeta(p)$ (for a suitable choice of the parameters) similar to what is found experimentally.

The GOY model can be seen as a severe truncation of the Navier-Stokes equations: it retains only one complex mode $u_{n}$ as a representative of all Fourier modes in the shell of wave numbers $k$ between $k_{n}=k_{0} 2^{n}$ and $k_{n+1}$.

Dynamical equations have the same qualitative structure of Navier-Stokes eqs., namely:

$$
\frac{d}{d t} u_{n}=N_{n}[\mathbf{u}]-\nu k_{n}^{2} u_{n}+f_{n}
$$

where $N[\mathbf{u}]$ are the inertial nonlinear terms (see below), while $\nu$ is the molecular viscosity and $f_{n}$ a suitable forcing term acting only at large scale introduced in order to reach a (statistical) stationary state.

The choice of the nonlinear term is dictated from the "locality assumption", i.e. only couplings with the nearest and next nearest shells are kept. In details the final eqs. are:

$$
\frac{d}{d t} u_{n}=i k_{n}\left(u_{n+1}^{*} u_{n+2}^{*}+b u_{n+1}^{*} u_{n-1}^{*}+c u_{n-1}^{*} u_{n-2}^{*}\right)-\nu k_{n}^{2} u_{n}+\delta_{n, n_{0}} f,
$$

where the the external forcing acts on a large scale $n_{0}$ and $b, c$ are two free parameters, but with the constraint $1+2 b+4 c=0$, used for changing the "dimensionality" of the system [28, 30], a popular choice which leads to results close to the $3 \mathrm{~d}$ turbulent phenomenology is $b=-1 / 4, c=-1 / 8$. Let 
us stress that this choice of the parameters corresponds to have both energy and helicity conservation for a shell scale ratio equal to 2 and that whenever one has these two invariants, for any shell ratio, one has anomalous scaling exponents.

At fixed molecular viscosity, $\nu$, the model develops a chaotic energy transfer to the small scales, with intermittent burst and deviation from K41 in good qualitative and quantitative agreement with what observed in true turbulent flow.

The natural question which we would like to analyze in this paper is whether a reversible system obtained from the original dissipative equation by imposing a global constraint will allow us to reproduce the standard results and whether one can learn something of more about the strong-dissipative and far-from equilibrium structure of the stationary statistics.

Therefore, following Gallavotti's suggestion we introduce a Lagrange multiplier $\alpha[\mathbf{u}]$ such as the eqs. of motion (8) preserve the total energy for any time, namely:

$$
\frac{d}{d t} u_{n}=N_{n}[\mathbf{u}]-\alpha[\mathbf{u}] k_{n}^{2} u_{n}+f_{n}
$$

In order to have the total energy $E=1 / 2 \sum\left|u_{n}\right|^{2}$ constant, one has to impose:

$$
\alpha[\mathbf{u}]=\frac{\Re\left(u_{0} f\right)}{\sum_{n} k_{n}^{2}\left|u_{n}\right|^{2}}
$$

Let us comment that in [5, 6] different versions of reversible - hydrodynamical equations for a flow have been proposed depending on which macroscopic observables one fixes by mean of the lagrangian multiplier. For example, equations similar to (9) but with $\alpha[\mathbf{u}]$ chosen such that the total energy dissipation is conserved could in principle be used as well. In our view, guided from the phenomenological behaviour of turbulent flows, we believe that the only realistic constraint one can safely impose to the equation is on the total energy. Constraining the total energy dissipation would put too much weight on the small scales statistics and would kill one of the most remarkable signature of turbulent flows: multifractal nature of energy dissipation.

The goal of our study is to understand how the system move away from the stationary and equilibrium state that one obtain when $f_{n}=0$ as soon as some energy pump and (perfect) energy sink are switched on $\left(f_{n}>0\right)$. 


\section{Numerical Results}

We first performed a benchmark numerical integration of a standard irreversible and dissipative GOY model with fixed viscosity and forcing. This integration allows us to fix "physical realistic" values for the observables of the reversible dynamics. Numerical evolution was given by a forth-order Runge-Kutta algorithm, for a GOY model with $N=23$ shells and a constant forcing on the first shell, $f=5 \times 10^{-3}(1+i)$. The integration time was several hundreds characteristic eddy turn-over times. We measured the structure functions and the average energy of the system. Afterwards, we integrated the reversible dynamical system keeping the total energy fixed to the mean value of the benchmark run. We kept all the other parameters of the model equal to those of the benchmark run, except for the value of the forcing which we let vary in order to switch continuously from a conservative equilibrium dynamics $(f=0)$ to a conservative non-equilibrium dynamics $(f>0)$.

In fig. 1 we show the behaviour of functions:

$$
\Sigma_{n, p}=\left\langle\left|\Im\left[u_{n} u_{n+1} u_{n+2}+\frac{1}{4} u_{n-1} u_{n} u_{n+2}\right]\right|^{p / 3}\right\rangle,
$$

for $p=2$ and for different values of the external forcing. $\Sigma_{n, p}$ represent the power $p / 3$ of energy flux from from shell $n$th to shell $(n+1)$ th divided by $k_{n}$. It is easy to show that in the inertial range the energy flux must be constant [27]. Therefore, it is natural to quantify the statistical properties of the energy transfer by measuring the scaling properties: $\Sigma_{n, p} \simeq k_{n}^{-\zeta_{p}}$. One can observe that in the limit with vanishing forcing there is equipartition between degrees of freedom. In this case the viscous term is very low - the viscosity is proportional to the value of the forcing - and we have essentially a truncated-Euler system with gaussian probability distributions of the shell variables $u_{n}$. When the forcing is increased there appears two different scaling ranges. In the first range (small $k$ 's) it is clearly distinguishable an energy cascade. In the second range (large $k$ 's) the energy is in equipartition among the degrees of freedom. Likewise the probability distribution functions of shells in equipartition have all the same functional non-gaussian form.

The range in which the energy cascade is observed is longer for higher forcing up to a critical forcing where the cascade range coincide with the inertial range of the original GOY model. 
For even higher forcing the system falls in a stable fixed point in which all the energy is concentrated in the first shell and all the other shell variables are zero.

We have checked that the cascade range is not due to finite size effects by performing a simulation with a larger number of shells $(N=28)$ and keeping all the others parameters constant.

The same behaviour has been obtained in a model in which the dissipation term has been put only in the dissipative range (the last 7 shells).

Likewise, we measured the scaling exponents of $\Sigma_{n, p}$ in the energy-cascade range, using the extended self similarity (ESS) in order to have more accurate fits. In the ESS one measures the behaviour of the structure functions of order $p$ versus the structure function of order 3 . In this way the scaling range is longer and measures of scaling exponents are more precise [20].

In fig. 2 we plotted the function of order 6 and in fig. 3 we show the behaviour of the scaling exponents compared to that of the GOY model. There is a clear intermittent energy cascade.

On heuristic grounds, Gallavotti [6] made the conjecture that a dissipative system and its time-reversible analog should be equivalent (in a statistical sense) if the time scale by which the viscosity reaches its mean value is shorter than the hydrodynamic time scales (i.e. the eddy turnover times). In this case the viscosity time-evolution would be confused with its average.

Although we did not find a quantitative agreement with the conjecture of Gallavotti the qualitative behaviour is in the right direction. We defined the characteristic time $\tau$ of $\alpha[\mathbf{u}]$ as the decaying time of the autocorrelation of its logarithm, that is defined by:

$$
C(\tau)=\frac{<x(t+\tau) x(t)>-<x(t)>^{2}}{<x(t)^{2}>-<x(t)>^{2}}
$$

where $x=\log (\alpha)$. We considered the logarithm of the viscosity function because the function itself has very large fluctuations (several order of magnitude larger than its average) and consequently the time average of its square has very long convergence times. We have found that in the case with the longer cascade range, i. e. in the system with statistical properties closer to the original GOY model, this characteristic time is shorter (see fig. 4) and consequently the time by which the viscosity reaches its average is shorter. 
Moreover let us stress that in this case $\alpha[\mathbf{u}]$ has a smaller mean square value, i.e. smaller fluctuations.

\section{Conclusions}

The statistical mechanics of fully developed turbulence has features which are very different from those of the usual equilibrium statistical mechanics of Hamiltonian systems. Recently, Gallavotti proposed a dynamical ensemble equivalence between hydrodynamical dissipative system, e.g. the NavierStokes equations, and time-reversible systems.

In this paper, we introduce and study a time-reversible dynamical system obtained, from a shell model for turbulence, changing the viscous term such as the energy is conserved. At small forcing values the system has statistical behaviours very close to those of a gas, i.e. energy equipartition and gaussian statistics. At increasing the forcing one has a non-equilibrium statistical stationary state with an energy cascade and anomalous scaling laws similar to those observed in turbulence.

The dynamical ensemble equivalence seems to be satisfied at least for the typical observables measurable in turbulent flows.

A relevant open problem remains for defining precisely the class of constraints allowed for the Dynamical Ensemble Equivalence hypothesis to hold.

\section{Acknowledgement}

This work was supported by INFN (Iniziativa Specifica Meccanica Statistica FI11) and INFM (Progetto di Ricerca Avanzato TURBO).

We thank F. Bonetto and G. Gallavotti for many stimulating discussions and continuous encouragement. We also thank L.P. Kadanoff and R. Pasmanter for useful discussions. 


\section{References}

[1] U. Frisch, Turbulence, (Cambridge University Press, Cambridge, UK, 1995).

[2] J. Sommeria, J. Fluid Mech., 170, 139 (1986).

[3] C. Beck and F. Schlögl, Thermodynamics of chaotic systems, (Cambridge University Press, Cambridge, UK, 1993).

[4] G. Gallavotti and E. G. D. Cohen, Phys. Rev. Lett. 74, 2694 (1995).

[5] G. Gallavotti, Phys. Lett. A 223, 91 (1996).

[6] G. Gallavotti, Dynamical Ensembles Equivalence in Fluid Mechanics, preprint in chao-dyn@xyz.lanl.gov, $\sharp 9605006$.

[7] Z. S. She and E. Jackson, Phys. Rev. Lett 70, 1255 (1993).

[8] R. H. Kraichnan, J. Atmos. Sci. 33, 1521 (1976).

[9] M. Briscolini and P. Santangelo, J. Fluid Mech. 270, 199 (1994).

[10] T. Bohr, M. H. Jensen, G. Paladin and A. Vulpiani, Dynamical Systems Approach to Turbulence, (Cambridge University Press, Cambridge, UK, 1997).

[11] L. Kadanoff, Physics Today, September 11 (1995).

[12] R. H. Kraichnan and D. Montogomery, Reports Prog. Phys. 43, 547 (1980).

[13] R. H. Kraichnan,J. Fluid Mech. 59, 745 (1973).

[14] J. Sommeria, C. Nore, T. Dumont and R. Robert, C. R. Acad. Sci. Paris, sèrie II 312, 999 (1991).

[15] J. Miller, Phys. Rev. Lett. 65, 2137 (1990).

[16] R. A. Pasmanter, Phys. Fluid, 6, 1236 (1994). 
[17] F. Bonetto, G. Gallavotti and P. Garrido, Chaotic Principle: an experimental test preprint in mp_arc@math.utexas.edu, $\sharp 96-182$.

[18] A. N. Kolmogorov, Dokl. Akad. Nauk SSSR 30, 9 (1941).

[19] C. Meneveau and K. R. Sreenivasan, J. Fluid Mech. 224, 429 (1991).

[20] R. Benzi, S. Ciliberto, R. Tripiccione, C. Baudet, C. Massaioli \& S. Succi, Phys. Rev. E 48, R29 (1993).

[21] R. Benzi, S. Ciliberto, C. Baudet and G. R. Chavarria, Physica D, 80, 385 (1994).

[22] R. M. Kerr, J. Fluid Mech., 211, 309 (1990).

[23] E.B. Gledzer, Sov. Phys. Dokl. 18, 216 (1973).

[24] M. Yamada and K. Ohkitani, Prog. Theor. Phys. 81, 329 (1989); J. Phys. Soc. Jpn. 56, 4210 (1987); Phys. Rev. Lett 60, 983 (1988); Prog. Theor. Phys. 79, 1265 (1988).

[25] M.H. Jensen, G. Paladin and A. Vulpiani; Phys. Rev. A 43, 798 (1991).

[26] L. Biferale, A. Lambert, R. Lima and G. Paladin; Physica D 80105 (1995).

[27] D. Pisarenko, L. Biferale, D. Courvoisier, U. Frisch and M. Vergassola, Phys. Fluids A65 2533 (1993).

[28] L. Kadanoff, D. Lohse, J. Wang and R. Benzi; Phys. Fluids 7617 (1995).

[29] R. Benzi, L. Biferale, R. Kerr and E. Trovatore, Phys. Rev. E 533541 (1996).

[30] L. Biferale and R. Kerr, Phys. Rev. E 52, 6113 (1995). 


\section{FIGURE CAPTIONS}

\section{- FIGURE 1:}

$\Sigma_{n, 2}$ vs $k_{n}$ in log-log scale obtained from an integration of the model with $N=23, K_{0}=6.25 \times 10^{-2}$ and different values of the forcing: $f=$ $5 \times 10^{-3}(1+i)$ (plus), $f=6 \times 10^{-3}(1+i)$ (squares), $f=8 \times 10^{-3}(1+i)$ (cross). Diamonds represent the results obtained in the benchmark integration (i.e., the original GOY model).

- FIGURE 2:

$\Sigma_{n, 6}$ vs $\Sigma_{n, 3}$ in log-log scale obtained for the system with the same parameters as in figure 1 and $f=8 \times 10^{-3}(1+i)$ (diamonds). The K41 line (dashed line) is also shown for comparison.

- FIGURE 3:

$\zeta_{p}$ vs $\mathrm{p}$ for the GOY model(diamonds) and for its reversible analog. Squares are obtained for $f=8 \times 10^{-3}(1+i)$ and pluses for $f=5 \times$ $10^{-3}(1+i)$. The anomalous exponents have been calculated using the ESS.

- FIGURE 4:

$C(\tau)$ vs $\tau$ for two different values of the forcing: $f=5 \times 10^{-3}(1+i)$ (solid line) and $f=8 \times 10^{-3}(1+i)$ (dashed line). 


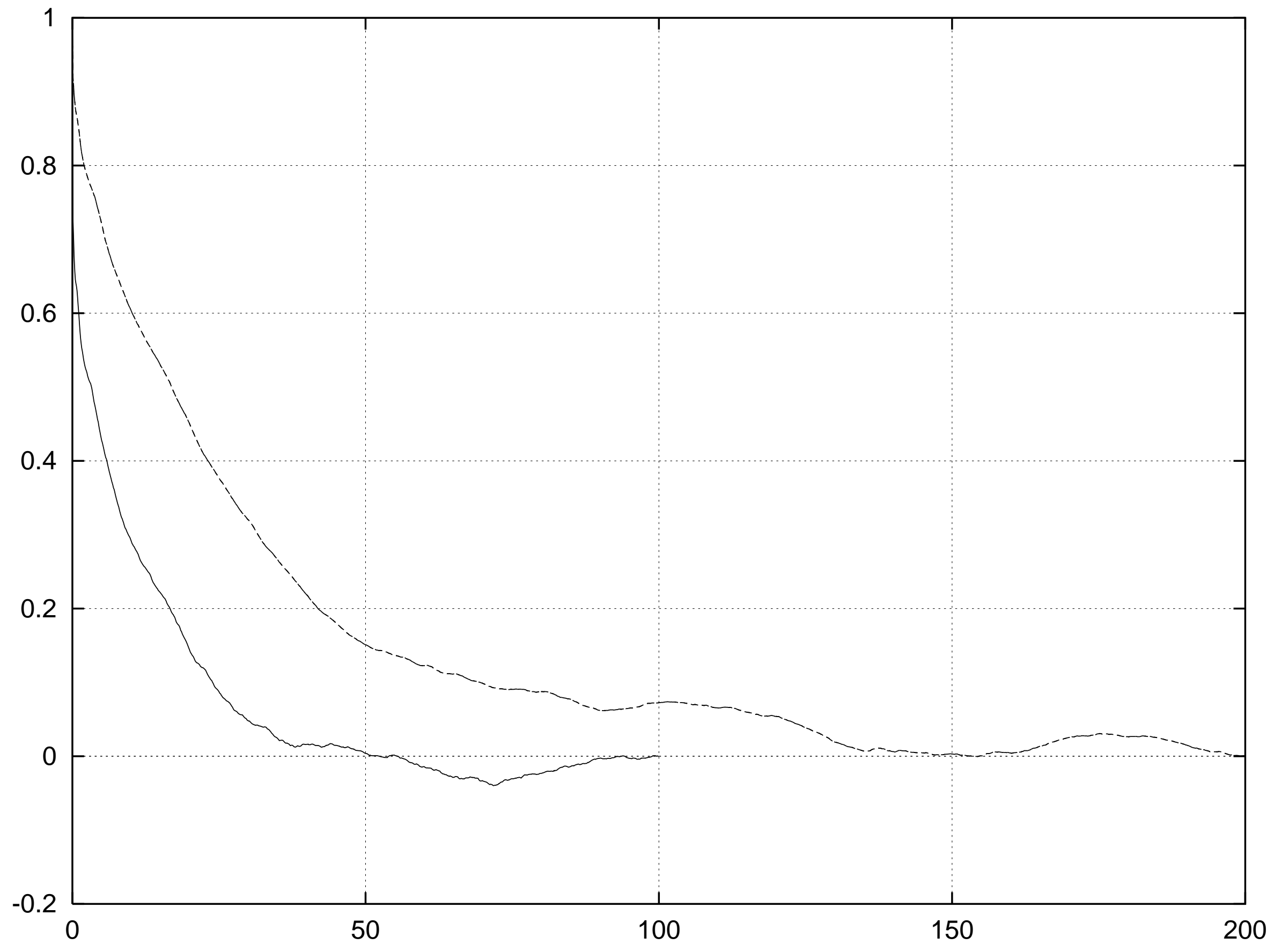




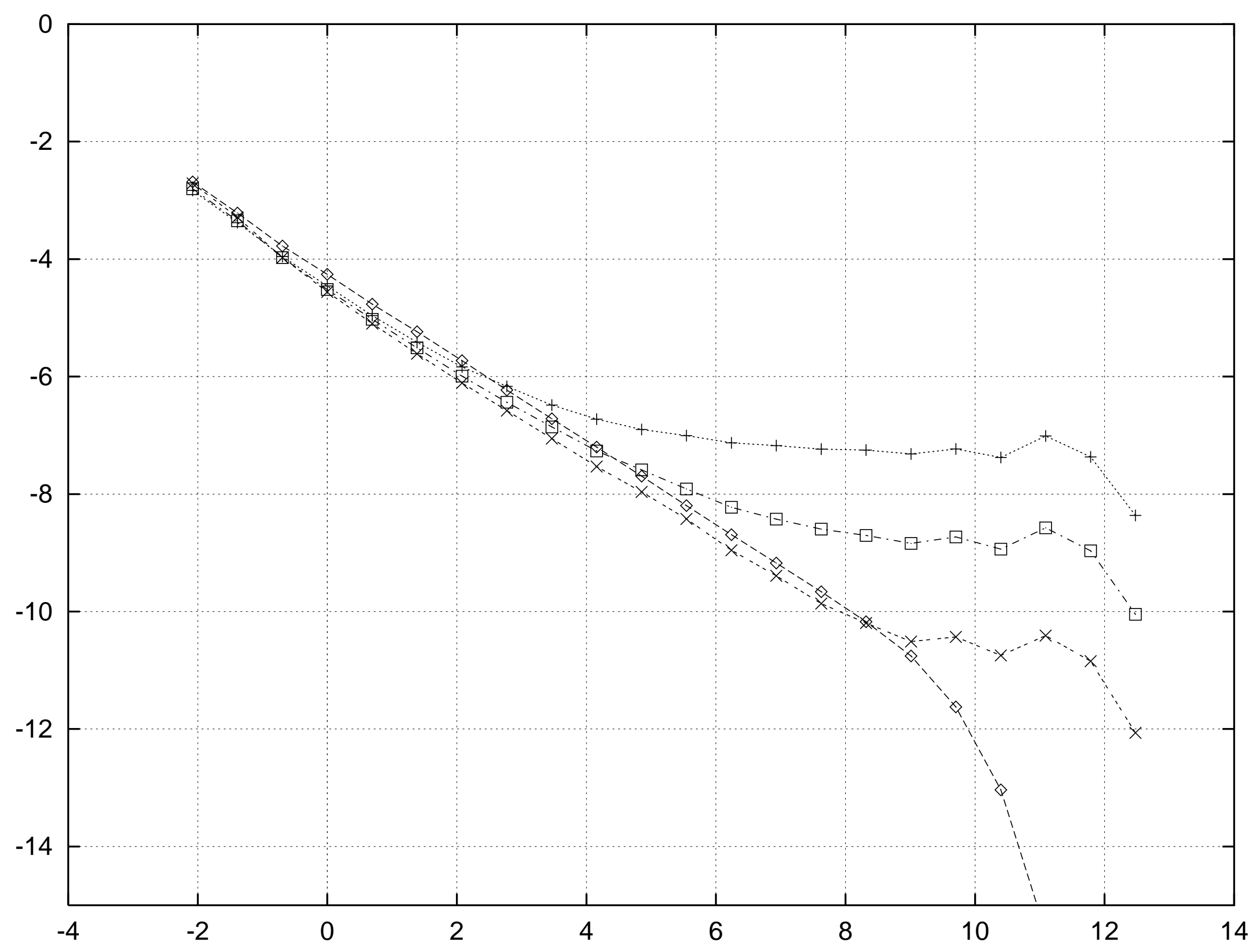




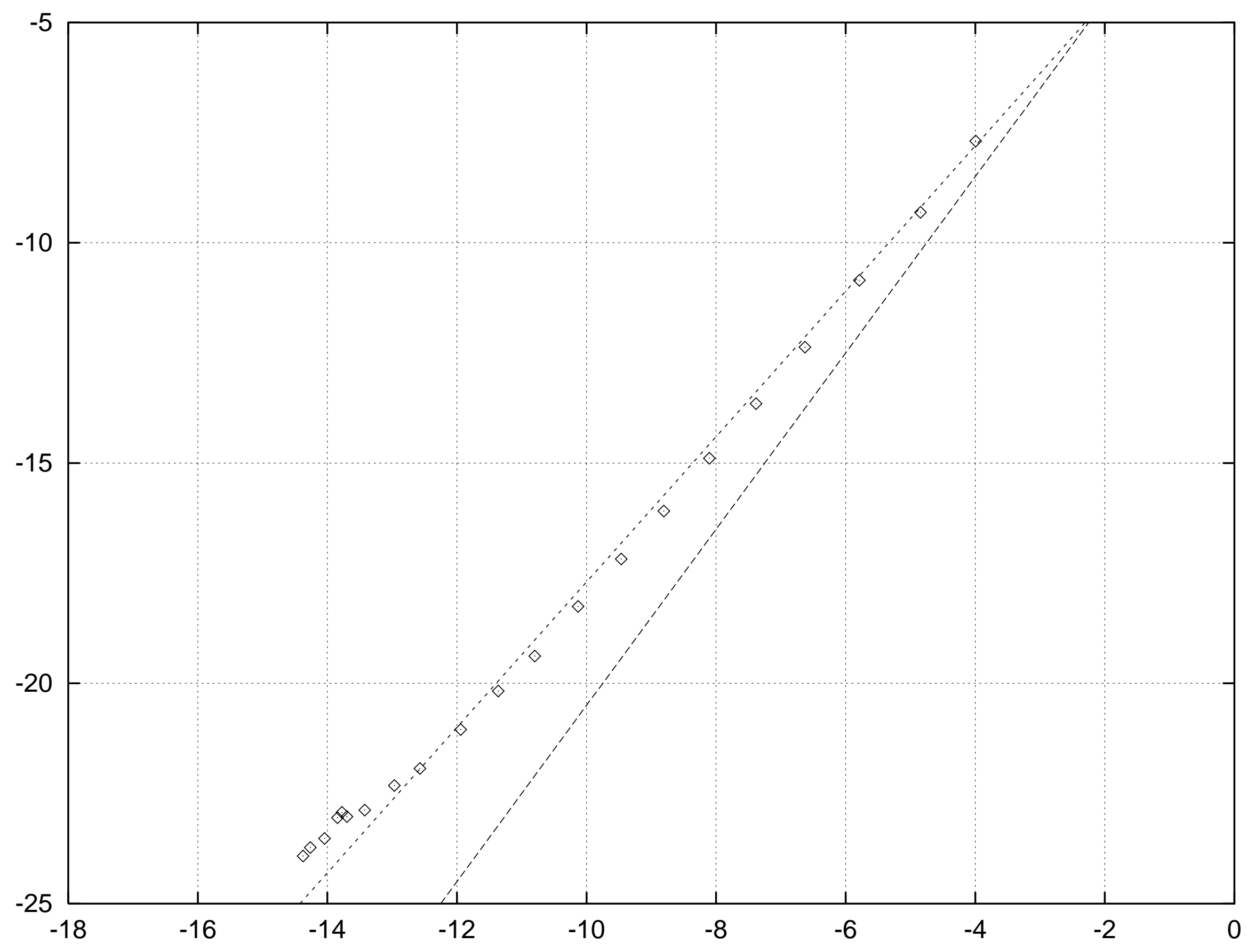




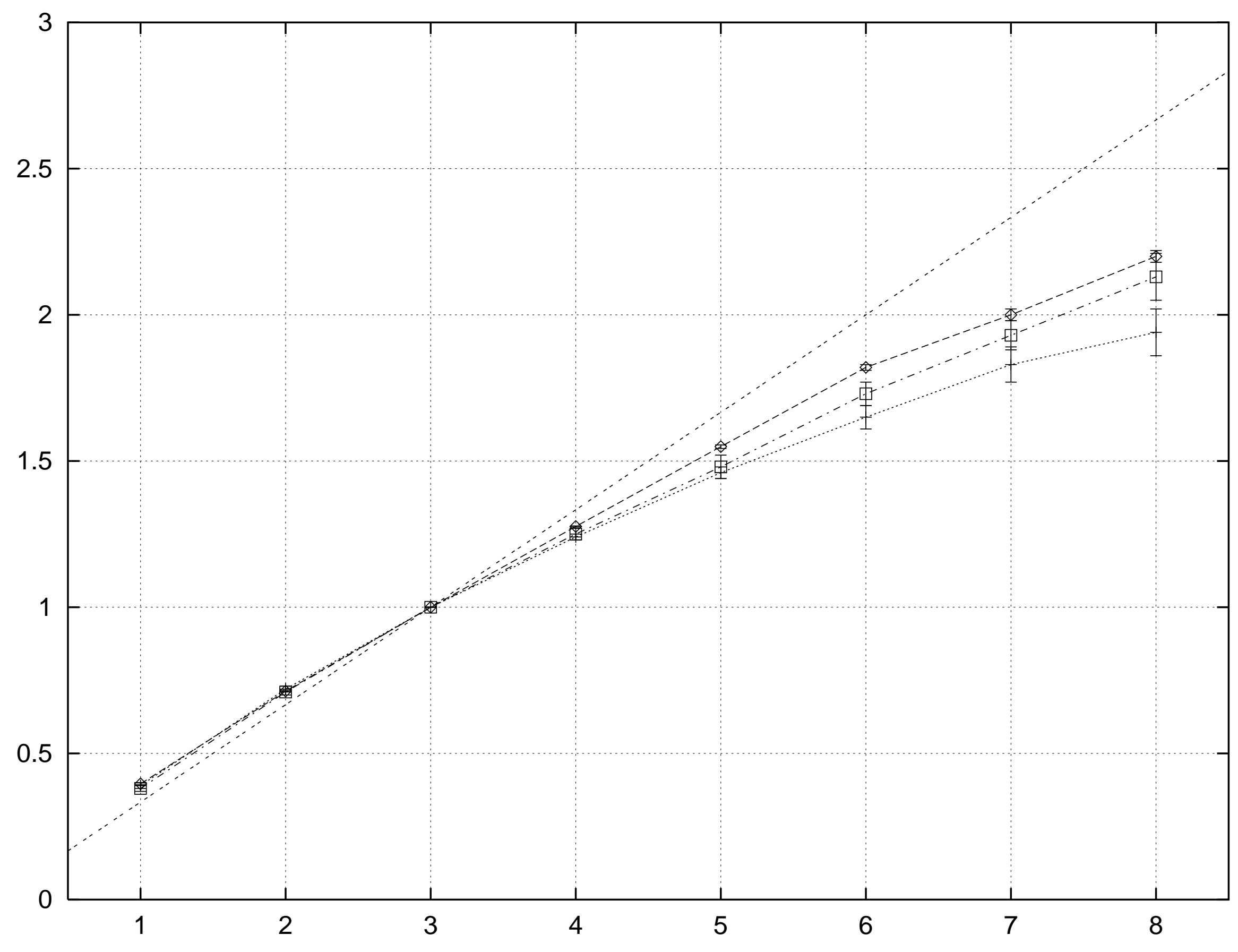

